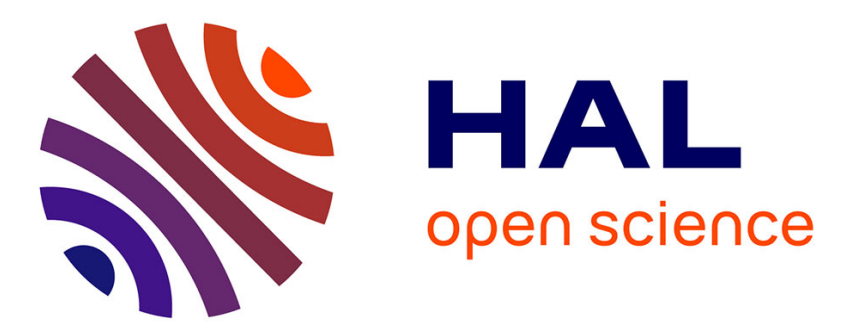

\title{
Recovering coefficients of the complex Ginzburg-Landau equation from experimental spatio-temporal data: two examples from hydrodynamics
}

Patrice Le Gal, Jean-Francis Ravoux, Elena Floriani, Thierry Dudok de Wit

\section{- To cite this version:}

Patrice Le Gal, Jean-Francis Ravoux, Elena Floriani, Thierry Dudok de Wit. Recovering coefficients of the complex Ginzburg-Landau equation from experimental spatio-temporal data: two examples from hydrodynamics. Physica D: Nonlinear Phenomena, 2003, 174, pp.114-133. 10.1016/S01672789(02)00686-3 . hal-00823553

\section{HAL Id: hal-00823553 https://hal.science/hal-00823553}

Submitted on 17 May 2013

HAL is a multi-disciplinary open access archive for the deposit and dissemination of scientific research documents, whether they are published or not. The documents may come from teaching and research institutions in France or abroad, or from public or private research centers.
L'archive ouverte pluridisciplinaire HAL, est destinée au dépôt et à la diffusion de documents scientifiques de niveau recherche, publiés ou non, émanant des établissements d'enseignement et de recherche français ou étrangers, des laboratoires publics ou privés. 


\title{
Recovering the coefficients of the Complex Ginzburg-Landau equation from experimental spatio-temporal data: Two examples from Hydrodynamics
}

\author{
P. Le Gal ${ }^{(1)}$, J.F. Ravoux ${ }^{(1)}$, E. Floriani ${ }^{(2)}$ and T. Dudok de Wit ${ }^{(3)}$ \\ ${ }^{(1)}$ IRPHE, 49 Av. F. Joliot-Curie, B.P. 146, Technopôle de Chateau-Gombert, 13384 Marseille, France \\ ${ }^{(2)}$ Centre de Physique Théorique, CNRS Luminy Case 907, 13288 Marseille cedex 9, France \\ ${ }^{(3)}$ LPCE-CNRS, 3A, av. de la Recherche Scientifique, 45071 Orléans cedex 2, France
}

April 26, 2002

\begin{abstract}
There are many examples where the description of the complexity of flows can only be achieved by the use of simple models. These models, obtained usually from phenomenological arguments, need in general the knowledge of some parameters. The challenge is then to determine the values of these parameters from experiments. We will give two examples where we have been able to evaluate the coefficients of the complex Ginzburg-Landau equation from space-time chaotic data applied to first a row of coupled cylinder wakes and then to wave propagation in the Ekman layer of a rotating disk. In the first case, our analysis is based on a proper decomposition of experimental chaotic flow fields, followed by a projection of the CGLE onto the proper directions. We show that our method is able to recover the parameters of the model which permits to reconstruct the spatio-temporal chaos observed in the experiment. The second physical system under consideration is the flow above a rotating disk and its cross-flow instability. Our aim is to study the properties of the wavefield through a Volterra series equation. The kernels of the Volterra expansion, which contain relevant physical information about the system, are estimated by fitting two-point measurements via a nonlinear parametric model. We then consider describing the wavefield with the complex Ginzburg-Landau equation, and derive analytical relations which express the coefficients of the Ginzburg-Landau equation in terms of the kernels of the Volterra expansion.
\end{abstract}




\section{Introduction}

Despite the large Reynolds numbers of the flows that occur in natural or industrial situations, their dynamical behaviour is very often dominated by the presence of large scale coherent structures. Classical examples can be found in atmospheric or oceanic vortex flows and there is some hope that simplified models might describe some of their complex or even chaotic spatio-temporal dynamics. Contrary to the situation encountered in many laboratory flows, one cannot study the responses of these flows to some control perturbations. In particular, when facing the problem of recovering the coefficients of some models, there is no way to excite these flows via periodic forcing as it is often the case to recover the dispersion relation of the waves which are involved in the solutions of the modelling equations. Therefore, it is necessary to develop some techniques which are able to adjust a non linear dynamical model to some measured data. This system identification thus leads to a problem of fitting a nonlinear differential equation to experimental data and is usually addressed in the field of nonlinear system identification [1]. This problem can be divided in three parts:

- model selection : determine what type of model should be fitted to the data,

- inference : estimate the model coefficients from the data,

- model validation : determine if the model indeed describes the observations adequately.

Unfortunately, there does not exist a unifying framework for describing nonlinear systems (like the Fourier transform for linear systems). One should therefore compare different approaches whenever possible. It should also be stressed that the criteria for obtaining a good model differ depending on whether one wants to make predictions, fit data, reproduce topological properties in phase space, etc. In the following, we present two techniques which enable us to estimate with a good accuracy the coefficients of the Complex Ginzburg Landau Equation in order to describe the space-time dynamics occuring in two instabilities of fluid flows. The first analysis is devoted to the coupled wakes downstream a row of cylinders and the second deals with the three-dimensionnal instability of a rotating disk boundary layer.

\section{Part 1: Spatio-temporal chaos generated by coupled wakes}

\subsection{Introduction}

It is known from experimental studies and numerical simulations (see the reviews by Zdrakovich [2] and Chang et al. [3]), that the wakes of bluff bodies placed side by side, can interact and create a large variety of phenomena. In the case of interest here, we analyze the space-time chaos created by the wakes of a row of 16 circular cylinders placed in a water tunnel perpendicular to an incoming flow. Figure 1 presents a snapshot of these 16 wakes made visible by dye injection through a small hole drilled at the rear front of each cylinder.

These cylinders possess a length of $200 \mathrm{~mm}$ and a diameter of $2 \mathrm{~mm}$. They are rigidly maintained in the wall of a water tunnel. The distance separating each cylinder axes is equal to four times their diameter and the Reynolds number Re of the flow is equal to 80 . Note that the separating distance has been chosen in accordance with previous results obtained on a pair of wakes by Peschard and Le Gal [4] and in such a way that the coupled wakes experience a spatio-temporal chaotic regime. In order to build space-time diagrams (512 time steps $\times 16$ space positions) which represent the dynamics of the family of wakes, we record a unique video line at the video frequency $(25 \mathrm{~Hz})$ and gather these lines together as presented in Figure 2. The acquisition line is situated $12 \mathrm{~mm}$ downstream the row of cylinders and the displacements of the dye streaks are recorded as a function of time (time unit is $0.04 \mathrm{~s}$ ). We can see on figure 2 the erratic appearance in space and time of amplitude holes [5].

It is known that the Bénard-von Karman wake of a cylinder placed in a flow appears via a Hopf bifurcation. Thus the oscillating flow can be modeled by a Stuart-Landau equation as it has been done by Provansal et al. in 1987 [6] or Dusek et al. in 1994 [7]: 


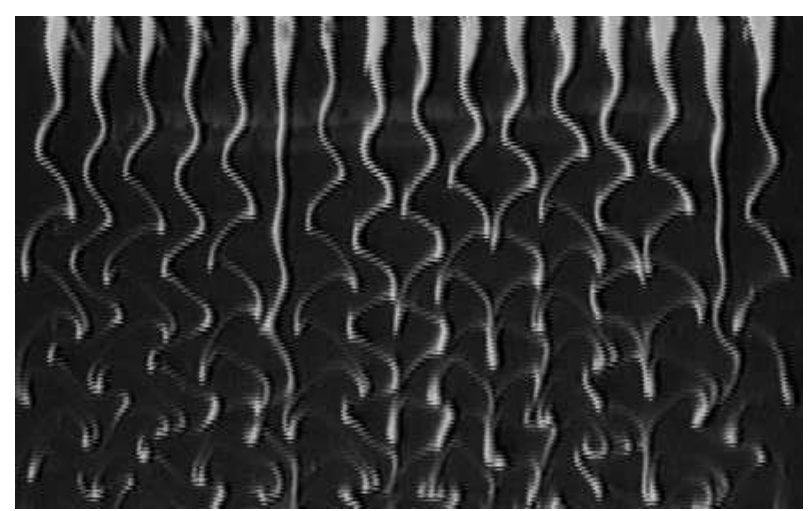

Figure 1: Snapshot of the 16 coupled wakes $(\mathrm{Re}=80)$ where amplitude holes can be observed on wakes 6 and 15.

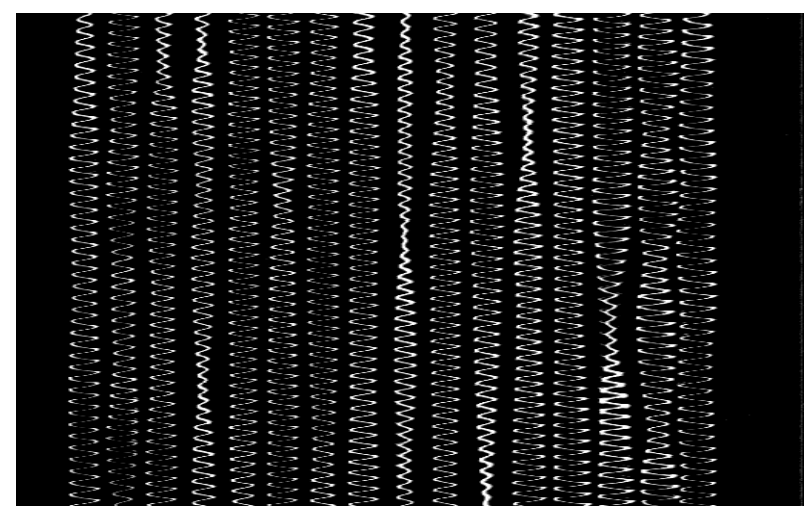

Figure 2: Space-time diagram, time is running downwards (10 seconds). The erratic appearance of amplitude holes is visible.

$$
d_{t} A(t)=\left(a_{r}+j a_{i}\right) A(t)-\left(l_{r}+j l_{i}\right)|A(t)|^{2} A(t)
$$

where $A$ represents an order parameter (for instance the transverse velocity at one position behind the cylinder). The complex coefficients $a=\left(a_{r}+j a_{i}\right)$ and $l=\left(l_{r}+j l_{i}\right)$ depend on the characteristics of the wake (aspect ratio or cross-section shape of the cylinder) and must be determined from experiments. Therefore, the coupled oscillators model that can be used to study the flow downstream the row of cylinders is a discrete version of the Complex Ginzburg-Landau equation (CGLE) (see Cardoso et al. $[8])$ :

$$
d_{t} A_{i}(t)=\left(a_{r}+j a_{i}\right) A_{i}(t)+\left(g_{r}+j g_{i}\right)\left(A_{i+1}(t)+A_{i-1}(t)-2 A_{i}(t)\right)-\left(l_{r}+j l_{i}\right)\left|A_{i}(t)\right|^{2} A_{i}(t)
$$

with the associated boundary conditions are $A_{0}(t)=A_{17}(t)=0$, where $A_{i}(t)$ is the complex amplitude of the wake of index $i$ and $g=\left(g_{r}+j g_{i}\right)$ is the linear coupling coefficient.

Since Eckhaus [9], most of the stability analysis of the Ginzburg-Landau equation have been carried out for the continuous case. The instability arises from a resonance mechanism between wave trains, and is called the Benjamin-Feir instability (or the sideband instability with modulations at $k \approx 0$ ). In particular, the well-know Newell's criterion [10] is related to the instability of any plane wave perturbed by a long wavelength modulation. More recently, Matkowsky and Volpert [11] considered perturbations 
of arbitrary wave numbers. Thus, they showed that destabilizations can occur through finite wave numbers perturbations and not only through homogeneous perturbations as it was classically studied. For a discrete system, the possible wave numbers of the waves are given by the number of oscillators and also by the boundary conditions applied on the array. The first known stability study for the discrete case has been performed by Willaime et al. in 1991 [12] with the wave numbers $q$ of the perturbations equal to 0 or $\pi$. These investigations have then been extended to all wave numbers for the basic solutions and to all wave numbers for the perturbations by Ravoux et al. [13].

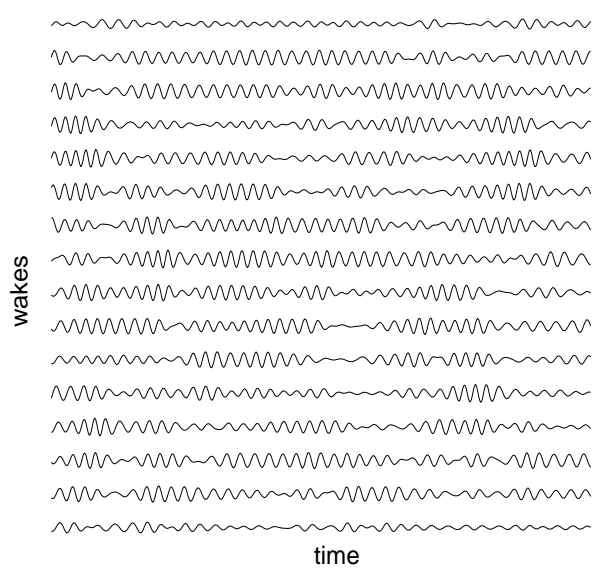

Figure 3: Numerical simulation of 16 coupled Landau oscillators $(a=1+20 j, g=1+3 j, l=1-1 j)$ with $10 \%$ noise added à posteriori.

\subsection{Recovering the coefficients of the model}

Contrary to what has been done for instance by Croquette et al. [14] in the study of non linear waves in Rayleigh-Bénard convection (where the dispersion relation was determined for each frequency by applying an external forcing), here we do not excite our hydrodynamical system but let it evolving in its natural spatio-temporal chaotic state. Therefore, our goal is to invert some measurements of space-time chaos in order to recover the coefficients of the model. Thus we need to solve linear systems of equations of the type $M_{1}=M_{2} x$, where $M_{1}$ and $M_{2}$ are matrices and $x$ an unknown vector. These matrices are made up from data and the vector $x$ consists in the coefficients $a, g$ and $l$ of the CGLE equation. The resolution is achieved via a least square method where the linear system is over-determined. Thus, the coefficient vector is such that the distance between $M_{1}$ and $M_{2} x$ is minimized in the associated phase space. The initial spatio-temporal field is a $512 \times 16$ matrix $A_{t i}$ that can be generated synthetically by numerical integration of CGLE as shown on Figure 3. However, as experimental data are corrupted by noise, we added $10 \%$ Gaussian noise à posteriori (at the end of the whole simulation) in order to test the robustness of our method [15]. Section 1.3 is thus devoted to test our methods on the results obtained from numerical simulations, where the coefficients are given à priori.

\subsection{Data from numerical simulations}

\subsubsection{Direct inversion}

In this case, the CGLE simply writes:

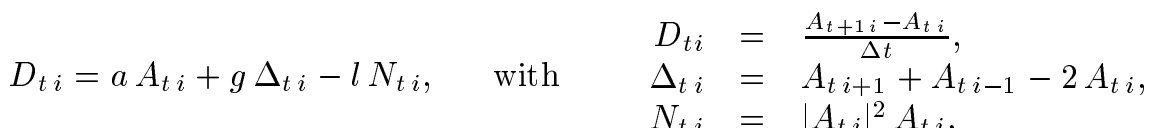



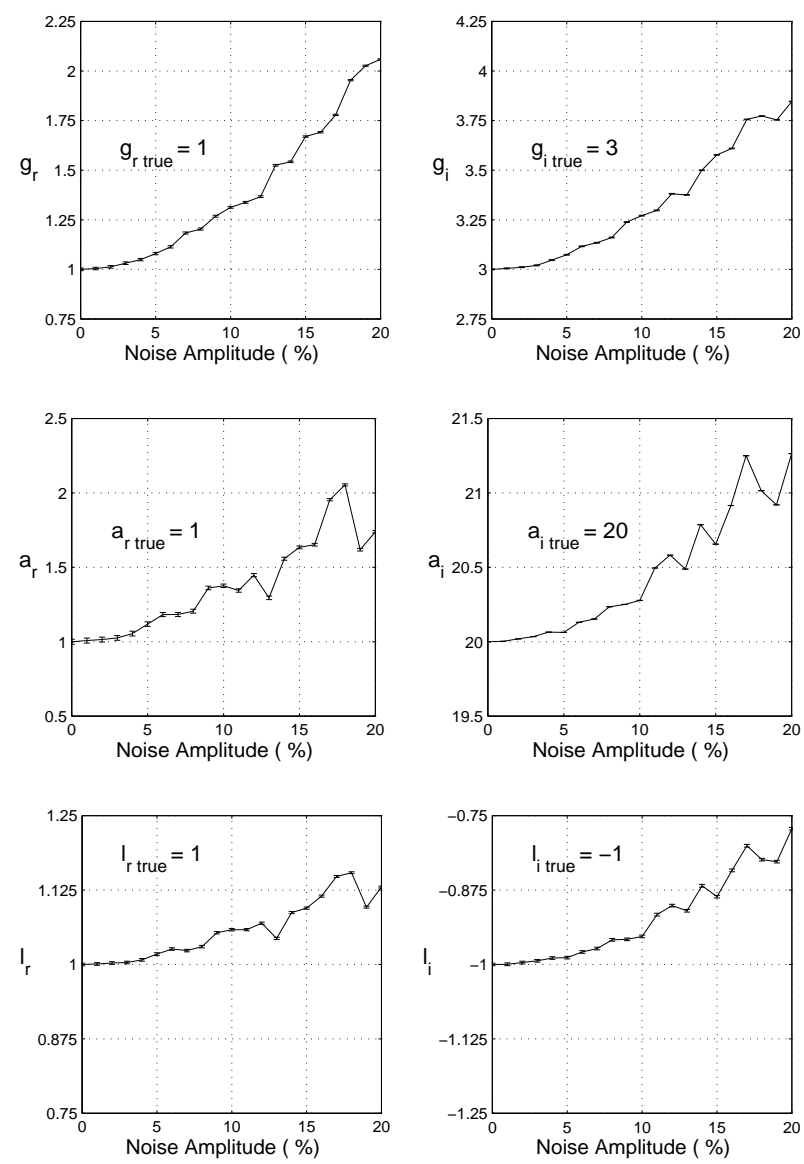

Figure 4: Direct Method, lack of accuracy of the inversion as a function of noise intensity.

where $\Delta t$ is the time unit given by the video acquisition rate. The goal of our work is thus to invert the algebraic system and to obtain the values of the coefficients $a, g$ and $l$. Similar direct inversion of chaotic spatio-temporal data series was done on the same problem by Fullana et al. [16], in a surface wave study by Gollub et al. [17], by Voss et al. for spatio-temporal measurements of binary-fluid convection [18], and in a reaction diffusion partial differential equation by Bär et al. [19].

As it can be seen on Figure 4, the presence of noise prohibits the recovery of the coefficients using this direct inversion method. The calculated values depend drastically on the noise intensity and depart strongly from the true values which have been chosen to compute the synthetic spatio-temporal data.

Even averaging the calculated coefficients on a great number of observation windows (typically 50 , corresponding to the total duration of the experimental data) does not cancel the influence of noise. Figure 5 shows in solid lines this departure from the true values and a rapid convergence to false values.

\subsubsection{Dispersion relation method}

In order to filter out the noise pollution which is reminiscent of any experiments, it is traditional to use the Fourier reciprocal space. Unfortunately, as it can be seen on the example of Figure 6, the Fourier power spectra computed on our experimental chaotic space-time diagrams do not permit a clear determination of the dispersion relation of the waves which are involved in the chaotic dynamics. Although, a more or less parabolic shape can be observed (note the negative curvature linked to the signs of $g$ and $l$ ), it is obvious that any fit of these spectra by a quadratic dispersion relation of Fourier modes deduced from 

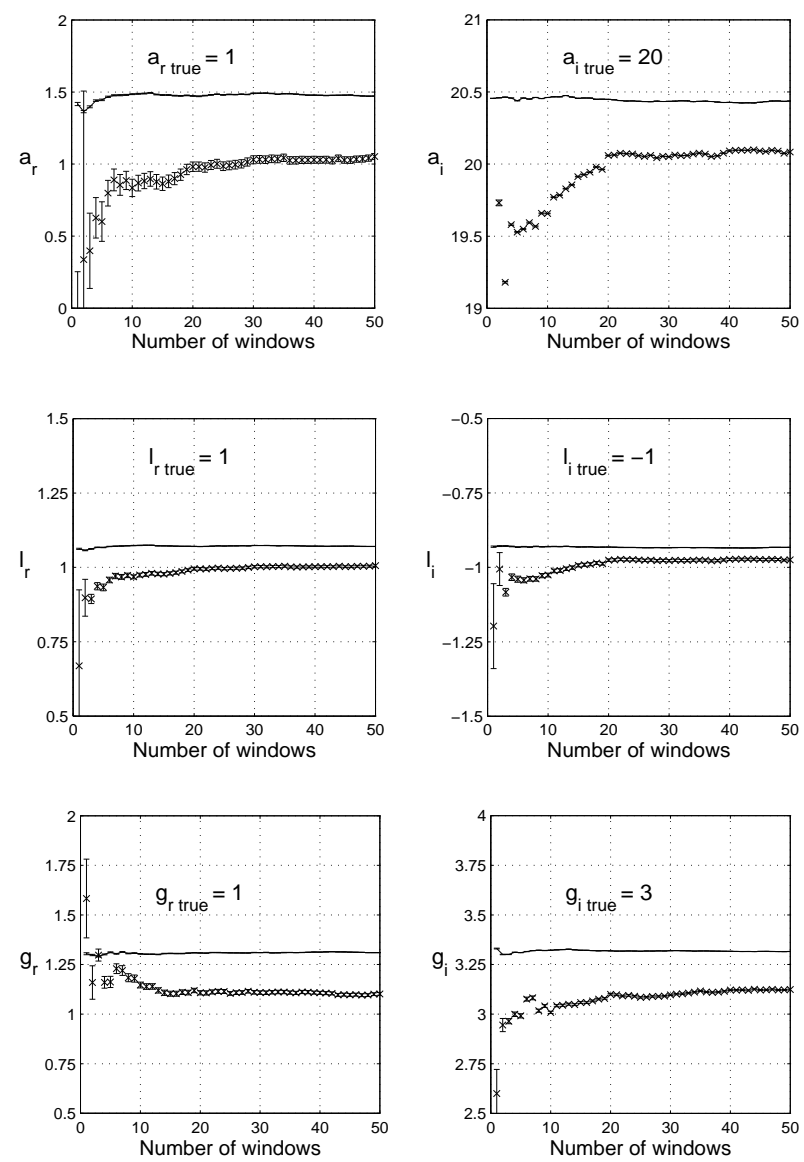

Figure 5: Simulated data: in solid line (-), lack of accuracy of the direct inversion as a function of observation windows. The crosses $(\mathrm{x})$ show better results obtained with the relation dispersion method. Error bars show the accuracy of the inversion.

the CGLE would lead to inaccurate coefficients. The reason why Fourier transforms are of little help for making a Galerkin projection, is because Fourier modes are not an adequate basis for representing the spatio-temporal dynamics of the wavefield. It would be more appropriate to seek a basis that exploits the properties of the data, giving what could be considered as "eigenmodes". Such a basis is provided by the Bi-Orthogonal Decomposition, also known as the Proper Orthogonal Decomposition [20].

Chauve and Le Gal proposed in 1992 [21] a method where we can filter the data and get a good characterization of the dispersion relation obtained by a Galerkin projection onto the proper modes of the Bi-Orthogonal Decomposition (BOD) [22]. Moreover, this method optimizes the total number of modes which are needed for the series reconstruction. The $N=16$ proper modes of the complex field $A_{t i}=A_{i}(t)$ are first calculated by diagonalization of the temporal correlation $(16 \times 16)$ matrix. Note that as the CGLE is a complex model, the first step of the method consists in complexifying the experimental data by the use of the Hilbert Transform. Then, the $N=16$ proper modes of the complex field $A_{t i}=A_{i}(t)$ are calculated by diagonalization of the temporal correlation $(16 \times 16)$ matrix and by the use of the projection relation:

$$
A_{t i}=\sum_{k=1}^{N} \alpha_{k} \psi_{k}(t) \bar{\phi}_{k}(i),
$$

where the overbar refers to the complex conjugation, $\psi_{k}$ the temporal mode and $\phi_{k}$ the spatial mode 


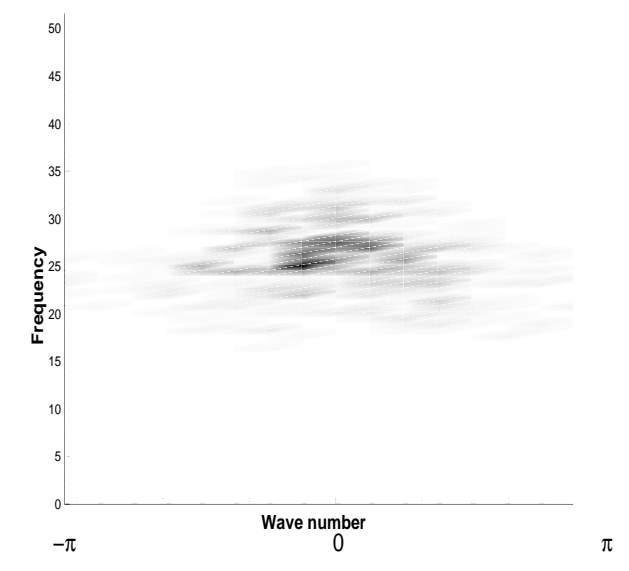

Figure 6: $(\mathrm{k}, \omega)$ Fourier Transform plane.

associated with the eigenvalue $\alpha_{k}$.
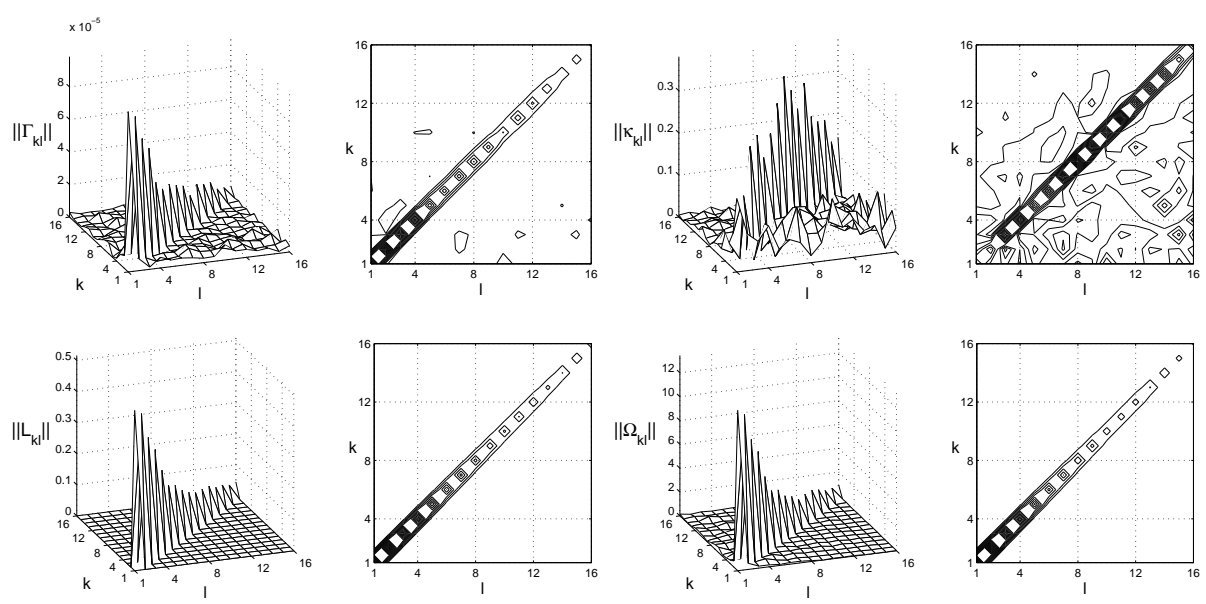

Figure 7: Simulated data: Matrices $\Gamma, \kappa, L$ and $\Omega$.

When the CGLE is projected onto the BOD modes, the following $(16 \times 16)$ matrices appear:

$$
\begin{array}{ll}
\Omega_{k l}=\left(\left(D_{t i} \phi_{i l}\right)^{*} \psi_{t k}\right)^{*}, & L_{k l}=\left(\left(A_{t i} \phi_{i l}\right)^{*} \psi_{t k}\right)^{*}, \\
\kappa_{k l}=\left(\left(\Delta_{t i} \phi_{i l}\right)^{*} \psi_{t k}\right)^{*}, & \Gamma_{k l}=\left(\left(N_{t i} \phi_{i l}\right)^{*} \psi_{t k}\right)^{*},
\end{array}
$$

and the Dispersion Relation linking modes $l$ and $k$ can then be written as (see pages 411-412 of [21]):

$$
\Omega_{k l}=a L_{k l}+g \kappa_{k l}-l \Gamma_{k l} .
$$

These matrices are therefore computed and the amplitudes of their entries are given in Figure 7. As it can be seen, they are essentially constituted by diagonal elements. The reason of this particular shape comes from the fact that the series are nearly pure sinusoidal functions which are themselves proportional to their own derivatives. Therefore, the inter-correlations (diagonal elements of the matrices) between the 
proper modes and their derivatives is higher than the cross-correlations. Using these diagonal elements, we can plot the projections of the dispersion relation (equation (4)) on the different directions. Figure 8 represents such a projection in the $3 \mathrm{D}$-space $\Omega_{r}, \Gamma_{i}, \kappa_{r}$. The linear relation between the different matrices is in complete accordance with equation (4). Therefore, determining the complex director vector of the dispersion relation plane in the space $(\Omega, \Gamma, \kappa)$ allows the calculation of the coefficients of the CGLE.

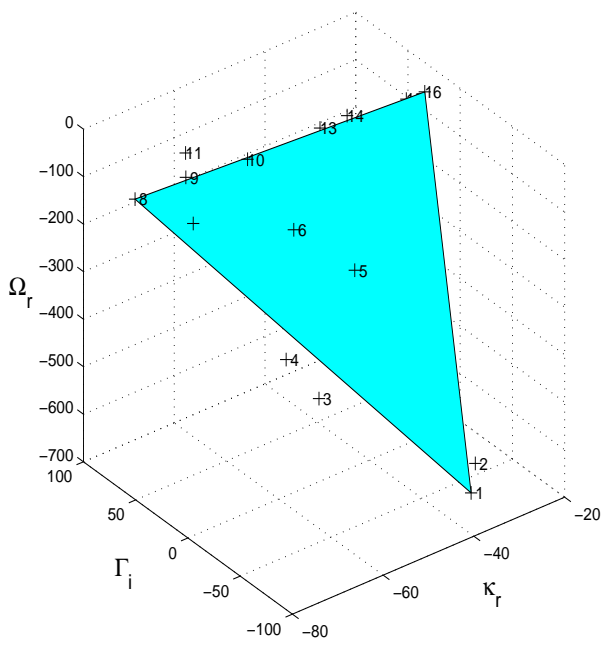

Figure 8: Simulated Data: Dispersion Relation in 3D space: $\Omega_{r}, \Gamma_{i}, \kappa_{r}$. In grey color is represented a portion of the dispersion relation plane.

Figure 5 presents the results (with the crosses $(\mathrm{x})$ ) of the calculations of the coefficients when averaging the obtained values on observation windows. We can observe that, although the convergence towards the true value is less rapid than the convergence of the direct method, the final result is satisfactory with an accuracy better than $7 \%$. For all these coefficients, the result is better than the one obtained using the direct method where some coefficients could depart from their true value of more than $50 \%$.

\subsection{Recovering the coefficients of the model from experimental data}

As our inversion method was successfully tested by our simulated data, we apply it to experimental space-time diagrams as the one presented in figure 2. Figure 9 shows the amplitudes of the entries of the four matrices $\Gamma, \kappa, L$ and $\Omega$. As it was previously observed on the simulated data study, most of the information is contained in the diagonal elements of the matrices. Therefore, only these diagonal elements, ordered by their index $l$ will be considered in the following. Then it can easily be seen on Figure 10 that most of the modes line up and validate the linearity of the dispersion relation (equation $(4))$.

To increase the accuracy of the linear regression, we then keep only the first twelve modes. The last four modes of the BOD, where the signal to noise ratio is observed to be less than $50 \%$ are thus neglected in the inversion process. The least square method then leads to the determination on the coefficients (average on 50 temporal windows):

$$
\begin{gathered}
a_{r}=-0.0534\left[(t)^{-1}\right], a_{i}=0.4747\left[(t)^{-1}\right], \\
g_{r}=-0.2396\left[(t)^{-1}\right], g_{i}=-2.7018\left[(t)^{-1}\right], \\
l_{r}=0.0567\left[(t)^{-1}(A)^{-2}\right], l_{i}=-0.0795\left[(t)^{-1}(A)^{-2}\right] .
\end{gathered}
$$

In fact, the relevant parameters of (2) are normalized and deduced from the latter, $\eta=\frac{g_{r}}{a_{r}}, c_{1}=\frac{g_{i}}{g_{r}}$ and $c_{2}=\frac{l_{i}}{l_{r}}: \quad \eta=4.48, \quad c_{1}=11.27, \quad c_{2}=-1.40$. 

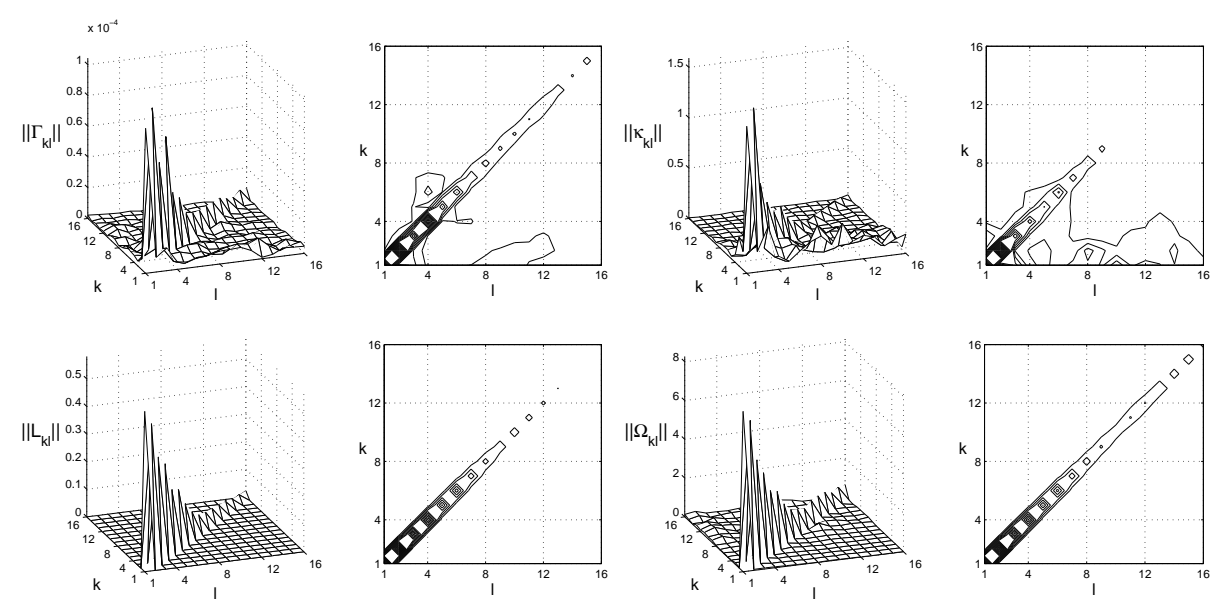

Figure 9: Experimental data: Matrices $\Gamma, \kappa, L$ and $\Omega$.
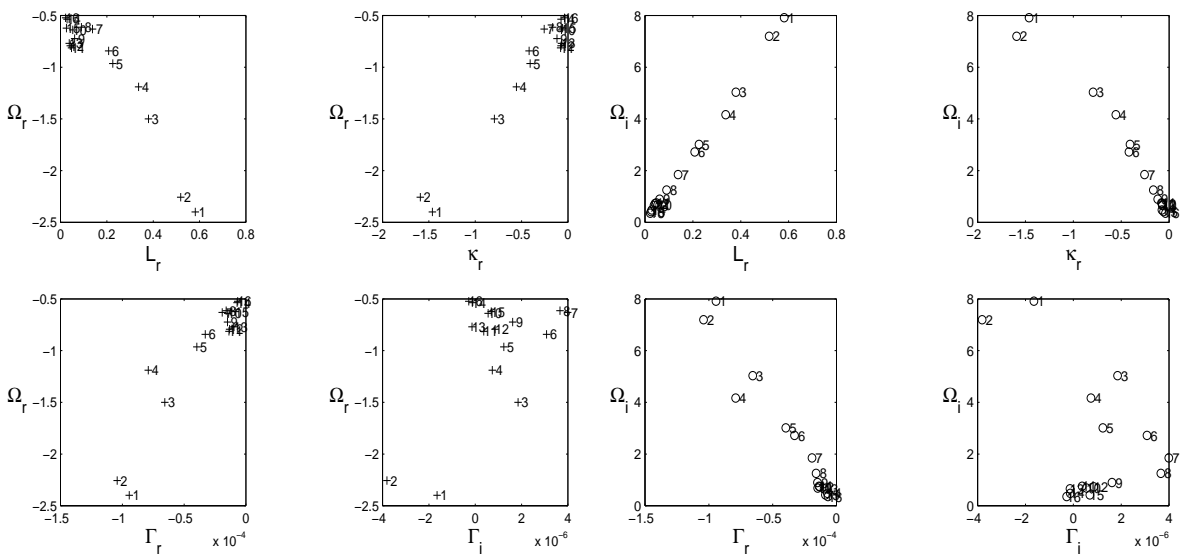

Figure 10: Experimental Data: Projection of $\Omega$ on some other directions. Each symbol is labeled by the index $l$ of the BOD modes.

which are coefficients with values in accordance with the values generally measured on wakes. Using these coefficients, it is easy now to test this validity by a numerical simulation of the CGLE. As it can be seen on figure 11, the reconstruction of the spatio-temporal dynamics shows a good agreement with the observed spatio-temporal chaotic behavior. In particular, the intermittent extinctions of oscillators (or amplitude holes) are recovered. Note that the main limitation of the BOD is that this method exploits second order moments of the data only. Because of this, it cannot properly capture deviations from a gaussian distribution, which are precisely a hallmark of nonlinear systems.

Another way to test our estimation of the coefficients of the model equation, is to compare simulated predictions with various experimental configurations and verify that the regime really experienced by our system can be indeed predicted. In our case, we changed the distance between the cylinders, so that the coupling coefficient between the wakes is varied. For distant cylinders, the wakes are poorly coupled and the phase between successive wakes is $\pi$. On the contrary, for short distance between cylinders, the wakes are strongly coupled and oscillate in an acoustic mode with a phase difference close to 0 (there can be a long wave spatial modulation along the row). As presented in figure 12, our numerical simulations recover correctly both this "in- phase" mode of the strong coupling case (note that the long wave modulations 


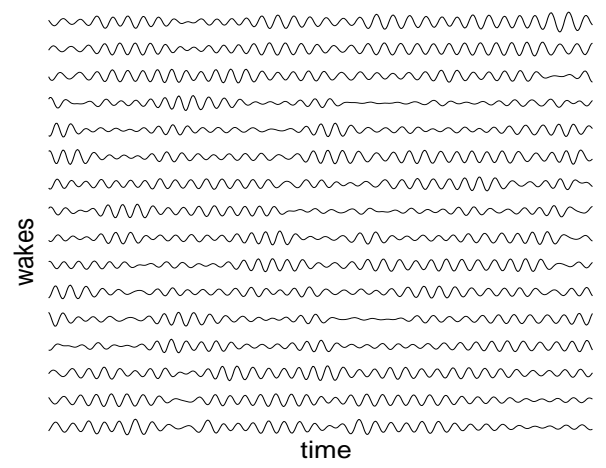

Figure 11: Space-time diagram of the 16 simulated coupled wakes with the coefficients obtained from the experiment.
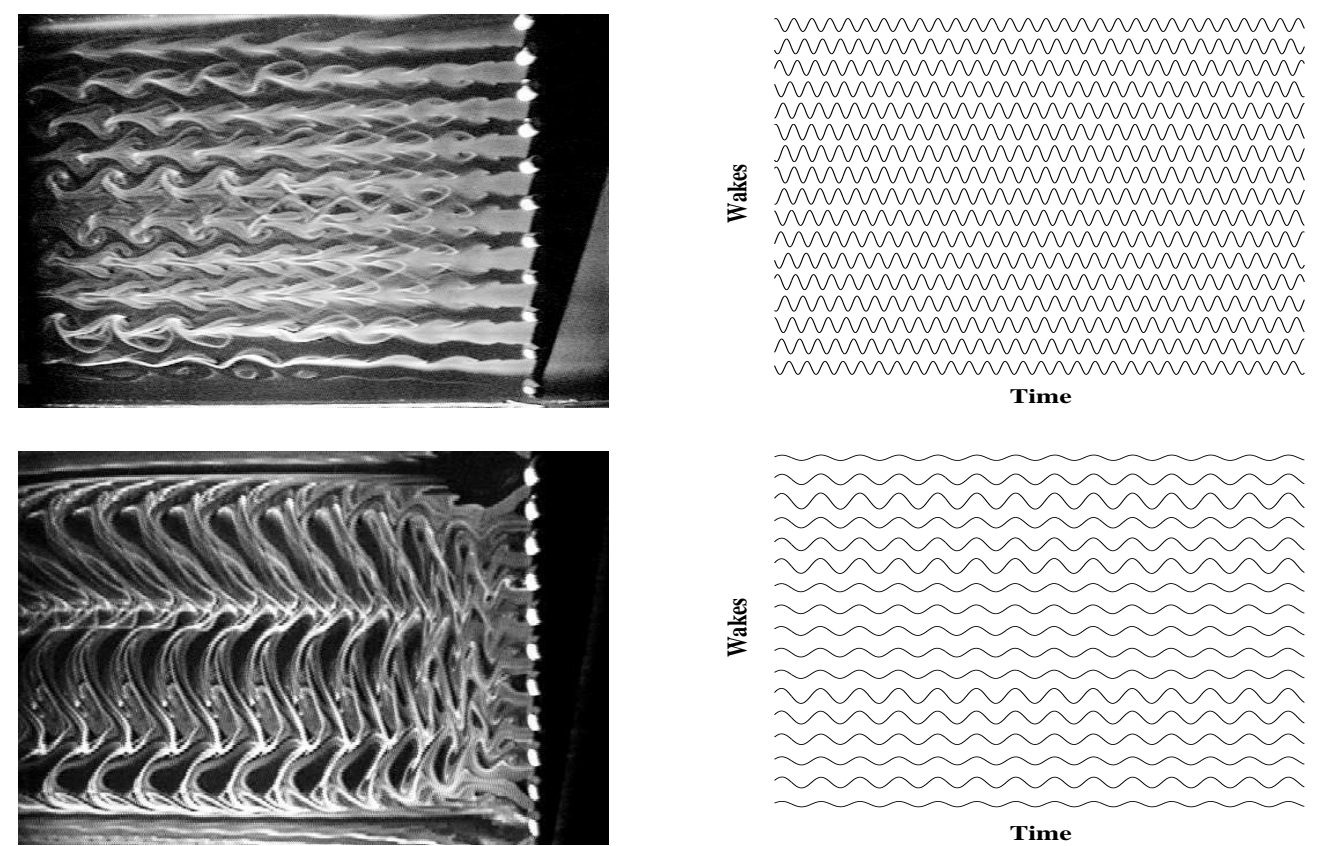

Figure 12: left: Visualizations of "anti-phase" (top) and "in-phase" modes (bottom, with long waves modulations) behind a row of cylinders. right: numerical simulations for weak and strong coupling, $(\eta=0.035$ (top) and 5 (bottom $))$, with the other coefficients obtained from experiments $\left(c_{1}=11.27, c_{2}=\right.$ $-1.40)$.

are also recovered) and the "anti- phase" mode of the weak coupling case [5].

\section{Part 2: Rotating disk flow instabilities}

\subsection{Introduction:}

Ekman was the first in 1905 [23], to formulate in a geophysical context, the mathematical expression of the velocity field of a rotating boundary layer. His analysis was based on the linearization of the fluid motion equations and the search for self-similar solutions. He supposed for that, that the fluid and the 


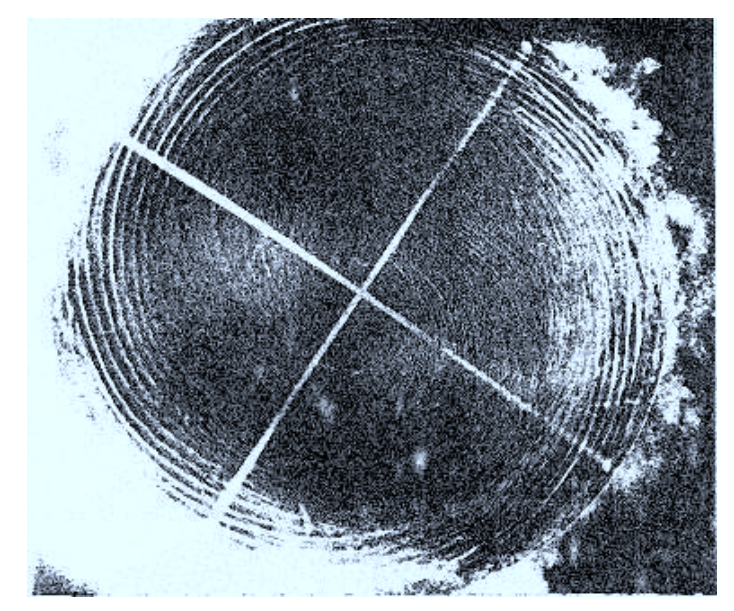

Figure 13: Type I instability waves in the Karman boundary layer.

disk have angular velocities very close one to the other. The self-similar solution he wrote takes the form of a spiral, now called the "Ekman Spiral", and is mainly localized in a thin boundary layer of depth $\delta$ nearby the rotating boundary $(\delta=\sqrt{\nu / \Omega})$. In 1921, Karman [24] this search for self-similar solutions to the full non linear case. Two types of instabilities (type I and type II) instabilities can appear in the rotating boundary layer. Type II instability corresponds to a destabilization by the combined effects of the forces due to the Coriolis and viscous effects. It produces waves which are rolled up in spirals in a contrary direction to the disk rotation. Stuart in 1955 [25] shows that type I instability is inviscid and comes from the presence of unstable inflection points in the radial velocity profiles. This instability also produces spiral waves but which are rolled up in the direction of rotation of the disk. Our experimental device is fully presented in Jarre et al. [26] and mainly consists of a $50 \mathrm{~cm}$ diameter horizontal disk, which is immersed in a water tank. Visualization of the type I waves is made possible when using a white dye as it is presented on figure 13. Around 30 wavelengths can be counted all around the disk.

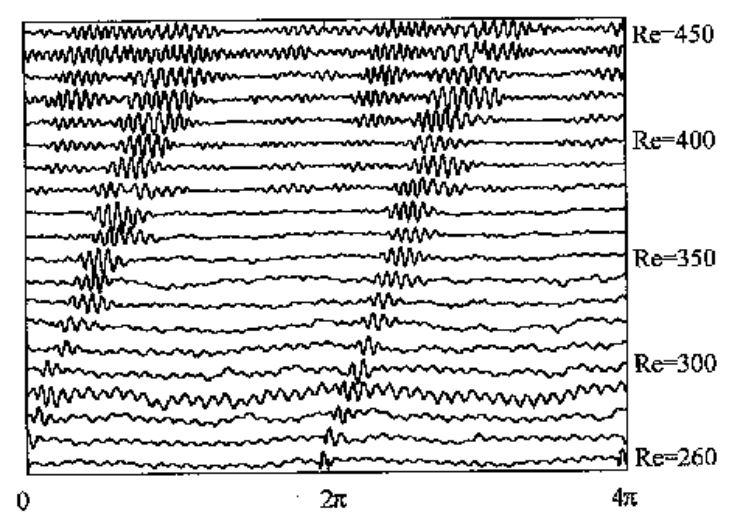

Figure 14: Wave packet shape widens as its amplitude grows.

Anemometric measurements of the waves generated by a small roughness element glued on the disk surface just under the linear threshold have been performed by the association of two anemometric probes, located at a distance of $8 \mathrm{~mm}$ one from the other, on a direction making an angle of 45 degrees from the radial direction. Figure 13 presents the shape of the wave pattern measured at different radial locations. 
The growth of its amplitude with Reynolds number (the radius) and the marginal stability curve which are obtained from Fourier analysis are given in figure 14. These measurements allowed in particular to estimate the azimuthal coherence length $\xi_{0 \theta}$ from the curvature of the marginal stability curve: a value around $1.2 \mathrm{~mm}$ was found [26] at the onset of the instability which occurs around $R e=280$. Then and before fully developed turbulence takes over for $R e \approx 510$ it was shown in Jarre et al. [27] that the non linear waves propagate with a well defined pattern and group velocity which justify an amplitude equation approach. However, the full determination of the values taken by the coefficients of the CGLE could not be obtained by the Fourier analysis developed in [27]. Next section will present our new technique based on Volterra series which allows such a determination [28].
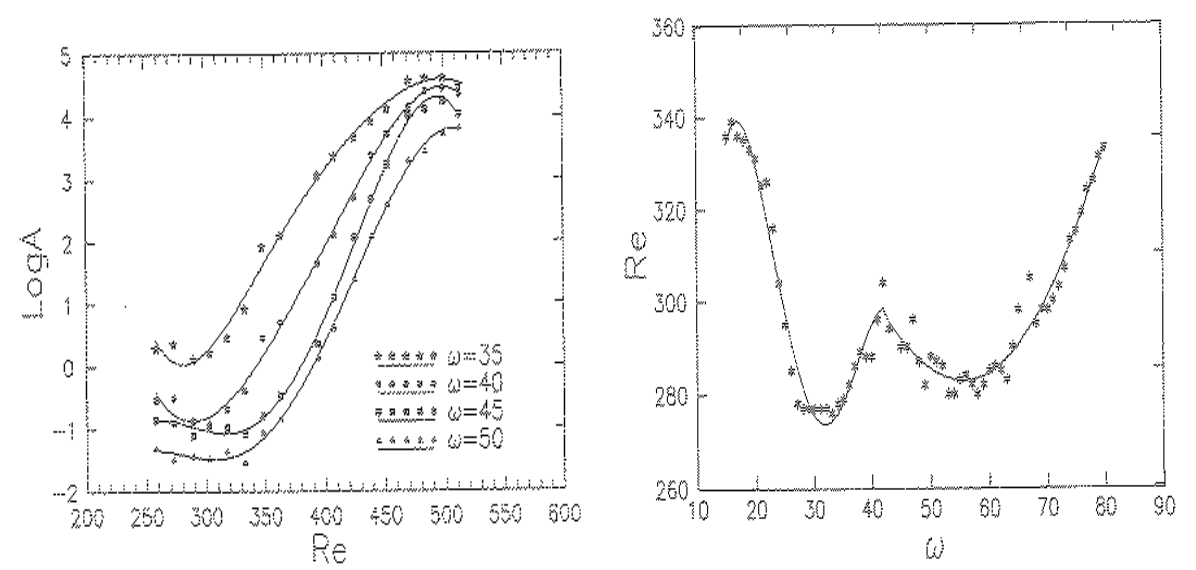

Figure 15: Growth of the waves along the radius and marginal stability curve. Note the second lobe corresponding to the growth of harmonics driven by non linear effects.

\subsection{Definition of the Volterra model}

We use here a different approach for inferring the coefficients of CGLE model. Instead of fitting this equation directly to the data (which would be an inductive approach), we first describe the data with a general class of models, based on Volterra series. If the underlying physics is indeed described by a CGLE model, then a direct mapping exists between the model coefficients and the Volterra kernels. In this way we can not only estimate the CGLE model coefficients, but also and more importantly, deduce whether this model is indeed correct.

Let $v(x, t, R e)$ be the azimuthal fluid velocity recorded at time $t$, position $x$ and Reynolds number $R e$. A general dynamical model for the wavefield amplitude is

$$
\frac{\partial v(x, t, R e)}{\partial x}=\mathcal{F}(v(x, t, R e)),
$$

where $\mathcal{F}$ is a continuous, nonlinear and time-invariant operator. We assume we can write $\mathcal{F}$ as a Volterra series [29], [30]:

$$
\begin{aligned}
\frac{\partial v_{i}(x, R e)}{\partial x} & =\sum_{k=0}^{\infty} g_{k}(R e) v_{i-k}(x, R e) \\
& +\sum_{k=0}^{\infty} \sum_{l=0}^{\infty} g_{k, l}(R e) v_{i-k}(x, R e) v_{i-l}(x, R e) \\
& +\sum_{k=0}^{\infty} \sum_{l=0}^{\infty} \sum_{m=0}^{\infty} g_{k, l, m}(R e) v_{i-k}(x, R e) v_{i-l}(x, R e) v_{i-m}(x, R e)+\cdots
\end{aligned}
$$


The wavefield $v$ is sampled at a constant rate, so a discrete version of the Volterra series is used here, with the notation $v_{i}(x, R e)=v\left(x, t=t_{i}, R e\right)$. The coefficients $g_{k}, g_{k, l}$ and $g_{k, l, m}$ are respectively called first, second and third order Volterra kernels. Furthermore, since we are dealing with nonlinearly interacting waves, it is appropriate to consider Fourier modes of the wavefield. The discrete Fourier transform in time gives:

$$
\begin{aligned}
\frac{\partial \hat{v}(x, \omega)}{\partial x} & =\Gamma(\omega) \hat{v}(x, \omega) \\
& +\sum_{\omega_{1}+\omega_{2}=\omega} \Gamma\left(\omega_{1}, \omega_{2}\right) \hat{v}\left(x, \omega_{1}\right) \hat{v}\left(x, \omega_{2}\right) \\
& +\sum_{\omega_{1}+\omega_{2}+\omega_{3}=\omega} \Gamma\left(\omega_{1}, \omega_{2}, \omega_{3}\right) \hat{v}\left(x, \omega_{1}\right) \hat{v}\left(x, \omega_{2}\right) \hat{v}\left(x, \omega_{3}\right) \\
& +\cdots
\end{aligned}
$$

where $\hat{v}(\omega)$ stands for the Fourier transform of $v_{i}$ at frequency $\omega$. The link between the Volterra kernels in Fourier space and their temporal counterparts is obviously:

$$
\begin{aligned}
\Gamma(\omega) & =\sum_{k=0}^{\infty} g_{k} e^{i \omega k}, \\
\Gamma\left(\omega_{1}, \omega_{2}\right) & =\sum_{k=0}^{\infty} \sum_{l=0}^{\infty} g_{k, l} e^{i\left(\omega_{1} k+\omega_{2} l\right)}, \\
\Gamma\left(\omega_{1}, \omega_{2}, \omega_{3}\right) & =\sum_{k=0}^{\infty} \sum_{l=0}^{\infty} \sum_{m=0}^{\infty} g_{k, l, m} e^{i\left(\omega_{1} k+\omega_{2} l+\omega_{3} m\right)},
\end{aligned}
$$

and so on for higher order kernels.

\subsection{Inversion of the Volterra model: determination of the Kernels}

We can give an estimation of the spatial derivative using the two-point measurements: as the probe separation $\Delta x$ is sufficiently small compared to the wave-length, we can write:

$$
\frac{\partial v_{i}(x, R e)}{\partial x} \approx \frac{v_{i}(x+\Delta x, R e)-v_{i}(x, R e)}{\Delta x} .
$$

The Volterra model may now be fully expressed into the more convenient framework of transfer functions:

$$
\begin{array}{rlr}
u_{i} & =v_{i}(x, R e) & \text { (the input) } \\
y_{i} & =v_{i}(x+\Delta x, R e) & \text { (the output) } \\
& =\sum_{k=0}^{n} \bar{g}_{k} u_{i-k}+\sum_{k=0}^{n} \sum_{l=0}^{n} \bar{g}_{k, l} u_{i-k} u_{i-l} \\
& +\sum_{k=0}^{n} \sum_{l=0}^{n} \sum_{m=0}^{n} \bar{g}_{k, l, m} u_{i-k} u_{i-l} u_{i-m} \\
& +\cdots+\varepsilon_{i},
\end{array}
$$

where $\varepsilon_{i}$ is the residual error that has to be minimised and where $\bar{g}$ is related to $g$ by

$$
g=\frac{\bar{g}}{\Delta x}
$$

The discrete transfer function (ref équation 4) is also known as a NX (Nonlinear with eXogeneous input) model. It is nothing but a nonlinear discrete mapping between the output and the input. 
The identification of nonlinear transfer functions from experimental data is a longstanding problem, since even for low order polynomials, the number of unknown coefficients can be huge. Many solutions have been developed for that purpose, see for example [1]. The key problem here is the selection of the model structure, i.e. the determination of kernels $g$ that significantly contribute to the wavefield dynamics. The procedure we have followed is detailed in [28].

For example, for $R e=387$, a least squares fit yields the model coefficients, which permit to reconstruct the signals as it is presented in figure 16 (Note that the residuals between the output and the predictions are barely visible on $16-\mathrm{b})$ :

$$
\begin{aligned}
y_{i}= & -0.7610 u_{i-8}+0.0032 u_{i-8} u_{i-1}^{2} \\
& -0.0105 u_{i-24}^{2} u_{i-3}+0.1022 u_{i} \\
& +0.0017 u_{i-23} u_{i-20} u_{i-8} \\
& -0.0115 u_{i-14} u_{i-3} u_{i-1} \\
& +0.0103 u_{i-23} u_{i-11} u_{i}+\varepsilon_{i}
\end{aligned}
$$

The most significant kernels are chosen among all possible combinations of linear, quadratic and cubic terms, with a memory (i.e. a number of delays $n$ ) equaling up to three wavefield periods. For all the Reynolds numbers of interest (Re going from 250 to 505), no higher order terms are needed to properly model the wavefield dynamics. This is an important result, since it justifies the truncation of the Volterra series at cubic terms, justifying the use of the CGLE as a model of wave propagation. More explicitely, let us emphazise that the truncation of the Volterra equation (2) at cubic terms is not made "a priori" but comes from the examination of the particular experimental data under consideration: for these data, higher order kernels in the series are negligible. As a consequence, the truncated Volterra eqution has to be related to the cubic CGLE.

\subsection{Relation to the complex Ginzburg-Landau equation}

The hydrodynamic field defining the wave packet, $v(x, t)$, can be written as:

$$
v(x, t)=A(x, t) e^{i k_{c} x-i \omega_{c} t}+c . c .
$$

where $A(x, t)$ is a complex function, slowly varying in space and time, and which obeys the CGLE:

$$
\tau_{0}\left[\frac{\partial A(x, t)}{\partial t}+V_{g} \frac{\partial A(x, t)}{\partial x}\right]=\mu A+\xi_{0}^{2}\left(1+i c_{1}\right) \frac{\partial^{2} A(x, t)}{\partial x^{2}}-l_{r}\left(1+i c_{2}\right)|A(x, t)|^{2} A(x, t)
$$

where

$$
\mu=\frac{R e-R e_{c}}{R e_{c}}
$$

$V_{g}$ is the group velocity, $\frac{\tau_{0}}{\mu}=a_{r}{ }^{-1}$ and $\xi_{0}^{2}=\eta$ give the characteristic time and length of the instability.

The general solution of a linear stability hydrodynamic problem can be expressed using a frequency and a wavenumber that verify a complex dispersion relation $\omega=\omega(k, R e)$. The coefficients $\tau_{0}, V_{g}, \xi_{0}, c_{1}$ are related to the Taylor expansion of the frequency $\omega(k, R e)$ near the critical threshold in the following way [31]:

$$
\begin{gathered}
\tau_{0}^{-1}=-\left.i R e_{c} \frac{\partial \omega}{\partial R e}\right|_{c}, \\
V_{g}=\left.\frac{\partial \omega}{\partial k}\right|_{c}, \\
\xi_{0}^{2}\left(1+i c_{1}\right)=\left.\frac{i \tau_{0}}{2} \frac{\partial^{2} \omega}{\partial k^{2}}\right|_{c},
\end{gathered}
$$



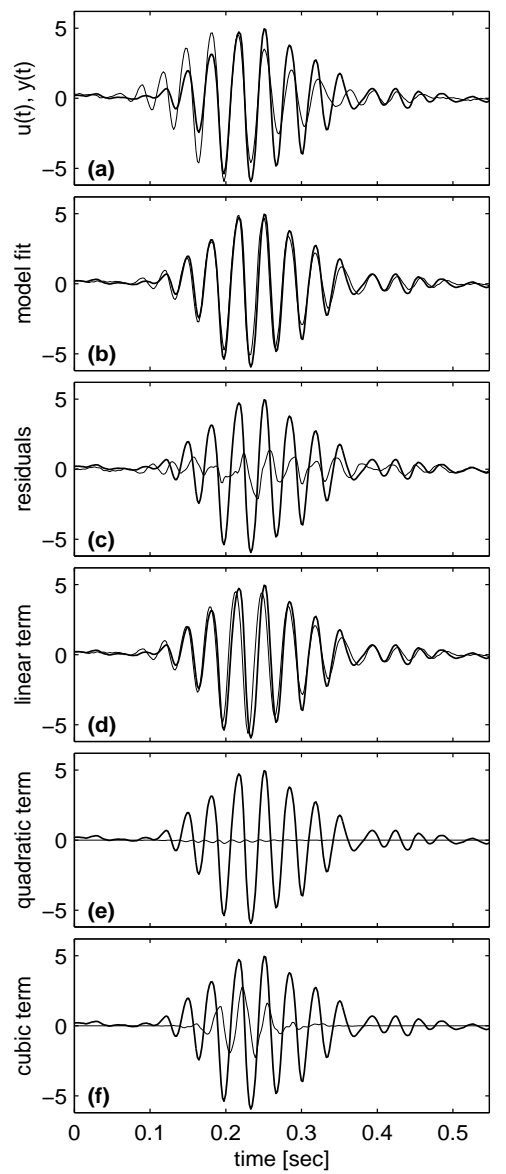

Figure 16: Excerpt of the wavefield amplitude at Re $=387$ showing from top to bottom (with the downstream probe always in bold) : (a) the measured in-and output, (b) the measured output and its prediction, (c) the measured output and the residuals, (d) the measured output and the linear constituent of the prediction, (e) the measured output and the quadratic constituent of the prediction, $(f)$ the measured output and the cubic constituent of the prediction. The measured signals are centered and reduced.

where $\left.\right|_{c}$ means that the partial derivatives are calculated at the critical point $R e=R e_{c}, k=k_{c}$. If the solution $A(x, t)$ is developed in a temporal Fourier series

$$
A(x, t)=\sum_{\nu} \hat{A}(x, \nu) e^{-i \nu t}
$$

it is easy to verify that the CGLE is equivalent to

$$
\begin{aligned}
0 & =i \nu \hat{A}(x, \nu)-\left.\frac{\partial \omega}{\partial k}\right|_{c} \frac{\partial \hat{A}(x, \nu)}{\partial x}+\left.\frac{i}{2} \frac{\partial^{2} \omega}{\partial k^{2}}\right|_{c} \frac{\partial^{2} \hat{A}(x, \nu)}{\partial x^{2}} \\
& -\left.i \frac{\partial \omega}{\partial R e}\right|_{c}\left(R e-R e_{c}\right) \hat{A}(x, \nu)-q \sum_{\nu_{1}+\nu_{2}+\nu_{3}=\nu} \hat{A}\left(x, \nu_{1}\right) \hat{A}\left(x, \nu_{2}\right) \hat{A}^{*}\left(x,-\nu_{3}\right),
\end{aligned}
$$

where $q$ is defined by

$$
q=\frac{l_{r}\left(1+i c_{2}\right)}{\tau_{0}}
$$


which can be compared to the Volterra equation (2), truncated at cubic terms (as it is justified in section $3.3)$ :

$$
\begin{aligned}
\frac{\partial \hat{v}(x, \omega)}{\partial x} & =\Gamma_{1}(\omega) \hat{v}(x, \omega) \\
& +\sum_{\omega_{1}+\omega_{2}=\omega} \Gamma_{2}\left(\omega_{1}, \omega_{2}\right) \hat{v}\left(x, \omega_{1}\right) \hat{v}\left(x, \omega_{2}\right) \\
& +\sum_{\omega_{1}+\omega_{2}+\omega_{3}=\omega} \Gamma_{3}\left(\omega_{1}, \omega_{2}, \omega_{3}\right) \hat{v}\left(x, \omega_{1}\right) \hat{v}\left(x, \omega_{2}\right) \hat{v}\left(x, \omega_{3}\right) .
\end{aligned}
$$

Near the critical threshold $R e=R e_{c}$, we write $v(x, t)$ in the form:

$$
v(x, t)=\left[A(x, t) e^{i k_{c} x-i \omega_{c} t}+B(x, t) e^{i 2 k_{c} x-i 2 \omega_{c} t}\right]+c . c .,
$$

with $A(x, t), B(x, t)$ slowly varying in space and time. Equation (12) gives then, for $\omega$ close to $\omega_{c}$ :

$$
\begin{aligned}
\frac{\partial \hat{A}\left(x, \omega-\omega_{c}\right)}{\partial x} & =\left[\Gamma_{1}(\omega)-i k_{c}\right] \hat{A}\left(x, \omega-\omega_{c}\right) \\
& +2 \sum_{\substack{\omega_{1}+\omega_{2}=\omega, \omega_{1} \simeq 2 \omega_{c}, \omega_{2} \simeq-\omega_{c}}} \Gamma_{2}\left(\omega_{1}, \omega_{2}\right) \hat{B}\left(x, \omega_{1}-2 \omega_{c}\right) \hat{A}^{*}\left(x,-\omega_{2}-\omega_{c}\right) \\
& +3 \sum_{\substack{\omega_{1}+\omega_{2}+\omega_{3}=\omega, \omega_{1} \simeq \omega_{2} \simeq-\omega_{3} \simeq \omega_{c}}} \Gamma_{3}\left(\omega_{1}, \omega_{2}, \omega_{3}\right) \hat{A}\left(x, \omega_{1}-\omega_{c}\right) \hat{A}\left(x, \omega_{2}-\omega_{c}\right) \hat{A}^{*}\left(x,-\omega_{3}-\omega_{c}\right),
\end{aligned}
$$

and for $\omega_{1} \simeq 2 \omega_{c}$ :

$$
\begin{aligned}
\frac{\partial \hat{B}\left(x, \omega_{1}-2 \omega_{c}\right)}{\partial x}= & {\left[\Gamma_{1}\left(\omega_{1}\right)-2 i k_{c}\right] \hat{B}\left(x, \omega_{1}-2 \omega_{c}\right) } \\
& +\sum_{\substack{\omega_{3}+\omega_{4}=\omega_{1} \\
\omega_{3} \simeq \omega_{4} \simeq \omega_{c}}} \Gamma_{2}\left(\omega_{3}, \omega_{4}\right) \hat{A}\left(x, \omega_{3}-\omega_{c}\right) \hat{A}\left(x, \omega_{4}-\omega_{c}\right)
\end{aligned}
$$

The adiabatic approximation leads then to:

$$
\hat{B}\left(x, \omega_{1}-2 \omega_{c}\right) \simeq-\frac{1}{\Re\left(\Gamma_{1}\left(\omega_{1}\right)\right)} \sum_{\substack{\omega_{3}+\omega_{4}=\omega_{1}, \omega_{3} \simeq \omega_{4} \simeq \omega_{c}}} \Gamma_{2}\left(\omega_{3}, \omega_{4}\right) \hat{A}\left(x, \omega_{3}-\omega_{c}\right) \hat{A}\left(x, \omega_{4}-\omega_{c}\right) .
$$

Substituting this expression in (14), we get

$$
\begin{aligned}
\frac{\partial \hat{A}\left(x, \omega-\omega_{c}\right)}{\partial x} & =\left[\Gamma_{1}(\omega)-i k_{c}\right] \hat{A}\left(x, \omega-\omega_{c}\right) \\
& +\sum_{\substack{\omega_{1}+\omega_{2}+\omega_{3}=\omega \\
\omega_{1} \simeq \omega_{2} \simeq-\omega_{3} \simeq \omega_{c}}} \Lambda\left(\omega_{1}, \omega_{2}, \omega_{3}\right) \hat{A}\left(x, \omega_{1}-\omega_{c}\right) \hat{A}\left(x, \omega_{2}-\omega_{c}\right) \hat{A}^{*}\left(x,-\omega_{3}-\omega_{c}\right)
\end{aligned}
$$

where $\Lambda$ is defined by:

$$
\Lambda\left(\omega_{1}, \omega_{2}, \omega_{3}\right)=-2 \frac{\Gamma_{2}\left(\omega_{1}, \omega_{2}\right) \Gamma_{2}\left(\omega-\omega_{3}, \omega_{3}\right)}{\Re\left(\Gamma_{1}\left(\omega-\omega_{3}\right)\right)}+3 \Gamma_{3}\left(\omega_{1}, \omega_{2}, \omega_{3}\right) .
$$


Differentiating equation with respect to $x$ we get:

$$
\begin{aligned}
\frac{\partial^{2} \hat{A}\left(x, \omega-\omega_{c}\right)}{\partial x^{2}}= & {\left[\Gamma_{1}(\omega)-i k_{c}\right]^{2} \hat{A}\left(x, \omega-\omega_{c}\right) } \\
+ & \sum_{\substack{\omega_{1}+\omega_{2}+\omega_{3}=\omega, \omega_{1} \simeq \omega_{2} \simeq-\omega_{3} \simeq \omega_{c}\\
}} \hat{A}\left(x, \omega_{1}-\omega_{c}\right) \hat{A}\left(x, \omega_{2}-\omega_{c}\right) \hat{A}^{*}\left(x,-\omega_{3}-\omega_{c}\right) \Lambda\left(\omega_{1}, \omega_{2}, \omega_{3}\right) \\
& {\left[\left(\Gamma_{1}(\omega)-i k_{c}\right)+\left(\Gamma_{1}\left(\omega_{1}\right)-i k_{c}\right)+\left(\Gamma_{1}\left(\omega_{2}\right)-i k_{c}\right)+\left(\Gamma_{1}\left(\omega_{3}\right)+i k_{c}\right)\right] . }
\end{aligned}
$$

Again, we keep only terms proportional to $A^{n}$ with $n \leq 3$. We will now replace the expressions (17) and (19) for $\frac{\partial \hat{A}(x, \nu)}{\partial x}$ and $\frac{\partial^{2} \hat{A}(x, \nu)}{\partial x^{2}}$ in the CGLE:

$$
\begin{aligned}
0= & i \hat{A}\left(x, \omega-\omega_{c}\right)\left\{\left(\omega-\omega_{c}\right)+\left.i \frac{\partial \omega}{\partial k}\right|_{c}\left[\Gamma_{1}(\omega)-i k_{c}\right]-\left.\frac{\partial \omega}{\partial R e}\right|_{c}\left(R e-R e_{c}\right)+\left.\frac{1}{2} \frac{\partial^{2} \omega}{\partial k^{2}}\right|_{c}\left[\Gamma_{1}(\omega)-i k_{c}\right]^{2}\right\} \\
+ & \sum_{\substack{\omega_{1}+\omega_{2}+\omega_{3}=\omega, \omega_{1} \simeq \omega_{2} \simeq-\omega_{3} \simeq \omega_{c}}} \hat{A}\left(x, \omega_{1}-\omega_{c}\right) \hat{A}\left(x, \omega_{2}-\omega_{c}\right) \hat{A}^{*}\left(x,-\omega_{3}-\omega_{c}\right)\left\{-\left.\frac{\partial \omega}{\partial k}\right|_{c} \Lambda\left(\omega_{1}, \omega_{2}, \omega_{3}\right)\right. \\
& \left.\left.+\left.\frac{i}{2} \frac{\partial^{2} \omega}{\partial k^{2}}\right|_{c} \Lambda\left(\omega_{1}, \omega_{2}, \omega_{3}\right)\left[\Gamma_{1}(\omega)+\Gamma_{1}\left(\omega_{1}\right)+\Gamma_{1}\left(\omega_{2}\right)+\Gamma_{1}\left(\omega_{3}\right)-2 i k_{c}\right)\right]-q\right\} .
\end{aligned}
$$

And identifying the terms, it comes:

$$
\begin{gathered}
V_{g}=i\left[\frac{\partial \Gamma_{1}}{\partial \omega}||_{c}^{-1},\right. \\
\tau_{0}=\left[\left.R e_{c} V_{g} \frac{\partial \Gamma_{1}}{\partial R e}\right|_{c}\right]^{-1}, \\
\xi_{0}^{2}\left(1+i c_{1}\right)=-\left.\frac{\tau_{0}}{2} V_{g}^{3} \frac{\partial^{2} \Gamma_{1}}{\partial \omega^{2}}\right|_{c}, \\
l_{r}\left(1+i c_{2}\right)=V_{g} \tau_{0}\left[2 \frac{\Gamma_{2}\left(\omega_{c}, \omega_{c}\right) \Gamma_{2}\left(2 \omega_{c},-\omega_{c}\right)}{\Re\left(\Gamma_{1}\left(2 \omega_{c}\right)\right)}-3 \Gamma_{3}\left(\omega_{c}, \omega_{c},-\omega_{c}\right)\right] .
\end{gathered}
$$

The imaginary part of the linear kernel $\Gamma(\omega)$ is directly related to the wavefield dispersion relation. The real part of $\Gamma(\omega)$ is related to the linear growth rate and is displayed in figure 17 together with the frequency $f_{c}$ of the fundamental mode. It can be checked that the zero growth rate curve is equivalent to the marginal stability curve displayed in figure 14. Positive values of the growth rate confirm the onset of the instability around $R e=300$, in good agreement with the Fourier analysis.

Using the values of Volterra kernels we get, near the linear threshold

$$
\begin{aligned}
V_{g} & =0.16 \pm 0.03 \mathrm{~m} \cdot \mathrm{s}^{-1} \\
\tau_{0} & =15.1 \pm 3.2 \mathrm{~ms} \\
\xi_{0} & =2.1 \pm 0.5 \mathrm{~mm} \\
c_{1} & =-0.47 \pm 0.28
\end{aligned}
$$

At this stage, we were not able to obtain statistically significant values for the coefficients $l_{r}$ and $c_{2}$ that are associated with the nonlinear term of the CGLE model. Extrapolating Extrapolating formulas (21) and (23) for $R e \neq R e_{c}$, we can get an estimation of the dependence of the group velocity $V_{g}$ and the diffusion length $\xi_{0}$ from Reynolds number. The resulting behaviour is consistent with the one presented in section 2.1 and the values calculated by Jarre et al. in 1996. Taking into account the angle of 45 


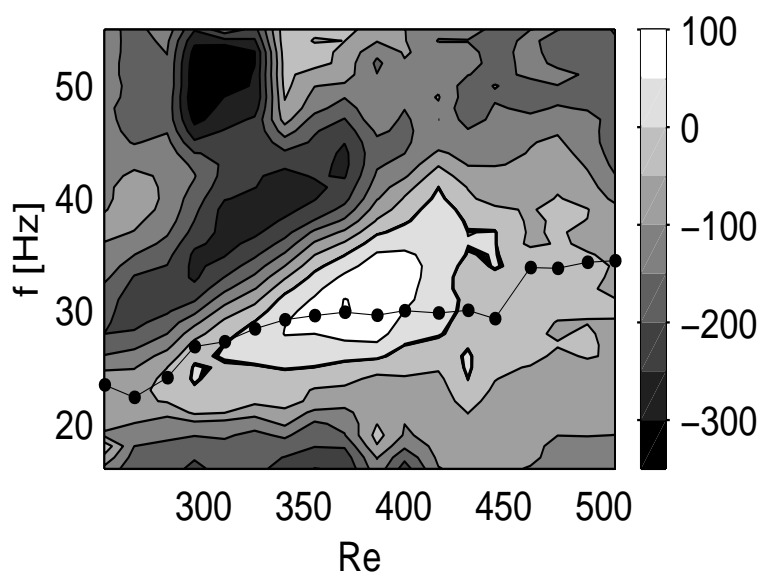

Figure 17: Real part of the linear Volterra kernel $\Gamma_{1}$ for various frequencies and Reynolds numbers. Superimposed on it is the frequency $f_{c}$ of the fundamental.

between the probes direction and the radial one, we find $\xi_{0 \theta}=1.4 \mathrm{~mm}$ which is close to the $1.2 \mathrm{~mm}$ obtained previously. Moreover, the transition from convective to absolute instability can also be checked, using the criterion obtained on the CGLE by Moon et al. in 1983 [31]:

$$
\eta_{a b s}=\frac{R e-R e_{c}}{R e_{c}}-\frac{V_{g}^{2} \tau_{0}^{2}}{4 \xi_{0}^{2}\left(1+c_{1}^{2}\right)} .
$$

$\eta_{a b s}$ gets positive for $R e=380$, which is in agreement Le Gal [32] where a transition towards a better spatio-temporal organisation of waves was detected for this value of the Reynolds number. Note that this threshold is lower to what was predicted by Lingwood [33] and thus does not correspond to the transition towards turbulence which is observed for a Reynolds number around 510 .

Clearly, these results are still open to improvements. The NX nonlinear model we have used is static in the sense that it cannot generate sustained oscillations if the input decays to zero. A significant reduction of the residuals and probably a better description of the topological properties of the system could be achieved by using a more general class of models, called Nonlinear Auto-Regressive Moving Average with eXogeneous input (NARMAX). This will be the object of a forthcoming publication.

\section{Conclusion}

In these studies, we have shown that measurements coming from image analysis or from anemometric signals and describing spatio-temporal chaotic propagation of waves, can be described by the Complex Ginzburg-Landau Equation.

In the first experiment which is devoted to coupled wakes, our concern was essentially to filter out the noise that pollutes the video images. Our method is based on the Bi-Orthogonal Decomposition (or Proper Orthogonal Decomposition) and leads to a generalized form of the dispersion relation of the waves. The noise is then easily removed as it is concentrated on modes having a high index: these modes are in fact poorly correlated. Therefore, the inversion problem is solved and the model coefficients can be extracted from the experimental data. Their values are in agreement with known properties of wakes and the reconstruction of chaotic space-time signals is then possible. Predictions of the dynamics have also been made by the use of the CGLE but for different coupling coefficients. These predictions have been favourably compared with experiments.

Our second analysis is devoted to the propagation of destabilizing waves in a rotating boundary layer. The measurements are performed synchronously at two slightly different locations above a rotating disk. 


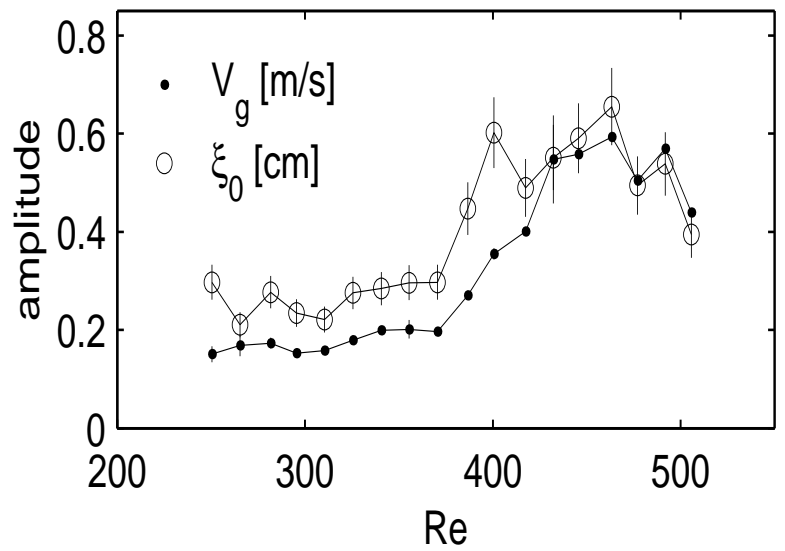

Figure 18: Group velocity $V_{g}$ projected along the probe separation vector, and diffusion length $\xi_{0}$. Error bars represent \pm one standard deviation.

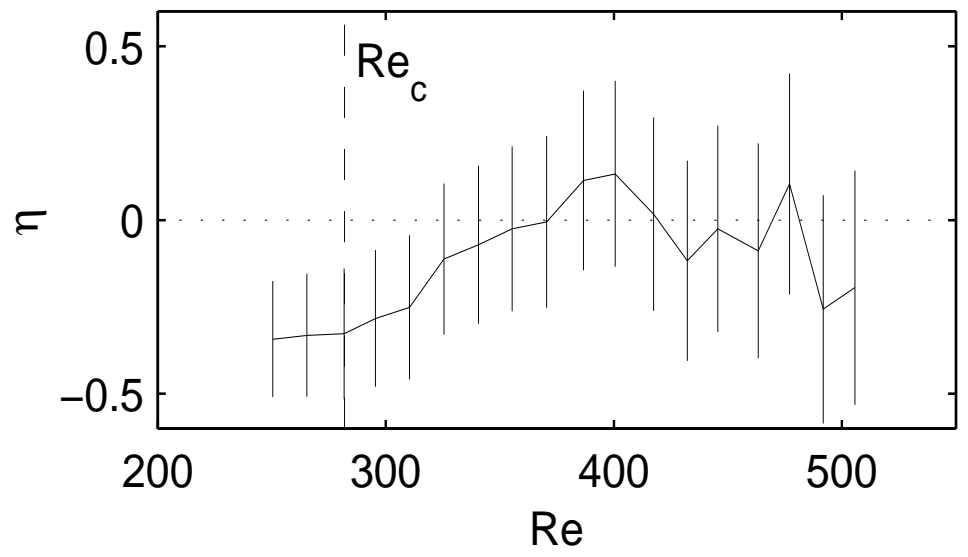

Figure 19: The flow becomes absolutely unstable when $\eta$ becomes positive.

Our analysis is based on Volterra series that allow the calculation of the linear properties of the wave trains (their non linear ones are currently under study). An analytical calculation that uses the elimination of fast harmonic modes made the connection between the Volterra kernels and the Ginzburg-Landau model. It is then possible to deduce the main properties of the wave propagation: growth rate, group velocity and coherence length. Moreover a transition from a convective instability to an absolute one has been discovered and explains in fact the growing coherence of the wave pattern that has been observed previously before its final transition to turbulence.

To conclude, let us remark that as both methods are based on general principles, they are not restricted to fluid mechanics and must hold for a large class of weakly nonlinear systems. 


\section{References}

[1] O. Nelles, Nonlinear System identification, Springer-Verlag, Berlin, 2001.

[2] M. Zdravkovich, Review of flow interference between two circular cylinders in various arrangements, Trans. ASME, J. Fluids Engng. 99 (1977) 618.

[3] K.S. Chang and C.J. Song, Interactive vortex shedding from a pair of circular cylinders in a transverse arrangement, Int. J. Numer. Meth. Fl. 11 (1990) 317.

[4] I. Peschard and P. Le Gal, Coupled wakes of cylinders, Phys. Rev. Lett. 77 (1996) 3122.

[5] P. Le Gal, Chaos spatio-temporel dans une chane de sillages couplés, C. R. Acad. Sci. 313, II (1991) 1499.

[6] M. Provansal, C. Mathis and L. Boyer, Bénard-von Karman instability: transient and forced regimes, J. Fluid Mech. 182 (1987) 1.

[7] J. Dusek, P. Le Gal and Ph. Fraunié, A numerical and theoretical study of the first bifurcation in a cylinder wake, J. Fluid Mech. 264 (1994) 172.

[8] O. Cardoso , H. Willaime and P. Tabeling, Short-wavelength instability in a linear array of vortices, Phys. Rev. Lett. 65 (1990) 1869.

[9] W. Eckhaus, Studies in Nonlinear Stability Theory, Springer, Berlin (1965).

[10] A.C. Newell, Envelope Equations, Lect. Appl. Math. 15 (1974).

[11] B.J. Matkowsky and V. Volpert, Stability of plane wave solutions of complex ginzburg-landau equations. Q. Appl. Math. 51 (2) (1993) 265.

[12] H. Willaime, O. Cardoso, and P. Tabeling, Coupled oscillators : an accurate model for describing the dynamics of lines of vortices, Eur. J. of Mech. B-Fluid 10 (2) (1991) 165.

[13] J. F. Ravoux, S. Le Dizes and P. Le Gal, Stability analysis of plane wave solutions of the discrete Ginzburg-Landau equation, Phys. Rev. E 61 (2000) 390.

[14] V. Croquette and H. Williams, Non-linear waves of the oscillatory instability of finite convective rolls, Physica D 37 (1989) 300.

[15] J. F. Ravoux and P. Le Gal, Bi-Orthogonal Decomposition analysis and reconstruction of spatiotemporal chaos generated by coupled wakes, Phys. Rev. E 58 (1998) R5233.

[16] J.M. Fullana, P. Le Gal, M. Rossi and S. Zaleski, Identification of parameters in an amplitude equation describing coupled wakes, Physica D 102 (1999) 35.

[17] D.P. Vallette, G. Jacobs and J.P. Gollub, Oscillations and spatio-temporal chaos of one-dimensional fluid fronts, Phys. Rev. E 55 (1997) 4274.

[18] H. U. Voss, P. Kolodner, M. Abel and J. Kurths, Amplitude Equations from Spatiotemporal BinaryFluid Convection Data, Phys. Rev. Lett. 83 (1999) 3422.

[19] M. Bär, R. Hegger and H. Kantz, Fitting partial differential equations to space-time dynamics, Phys. Rev. E 59 (1999) 337.

[20] P. Holmes, J. L. Lumley, and G. Berkooz, Turbulence, Coherent Structures, Dynamical Systems and Symmetry, Cambridge University Press, Cambridge, 1996.

[21] M.P. Chauve and P. Le Gal, Complex bi-orthogonal decomposition of a chain of coupled wakes, Physica D, 58 (1992) 407. 
[22] N. Aubry, R. Guyonnet and R. Lima, Spatio-temporal analysis of complex signals: theory and applications, J. Stat. Phys. 64 (1991) 683.

[23] V. W. Ekman, On the influence of the Earth's rotation on ocean currents, Arkiv. Mat. Astr. Fys. 2 (11) (1905) 1.

[24] T. Von Karman, Über laminare und turbulente Reibung, Z. Angew. Math. Mech. 1 (1921) 233.

[25] N. Gregory, J. T. Stuart and W. S. Walker, On the stability of three-dimensional boundary layers with application to the flow due to a rotating disk, Phil. Trans. R. Soc. London 248 (A) (1955) 155.

[26] S. Jarre, P. Le Gal and M. P. Chauve, Experimental study of rotating disk flow instability. I. Natural flow, Phys. Fluids 8 (1996) 496.

[27] S. Jarre, P. Le Gal and M. P. Chauve, Experimental study of rotating disk flow instability. II. Forced flow, Phys. Fluids 8 (1996) 2985.

[28] E. Floriani, T. Dudok de wit and P. Le Gal, nonlinear interactions in a rotating disk flow: From a Volterra model to the ginzburg-Landau equation. Chaos, vol 10, 4 (2000) 834.

[29] M. Schetzen, The Volterra and Wiener theories of nonlinear systems, Wiley, Chichester, N.Y. (1980).

[30] S. A. Billings, Identification of nonlinear systems - a survey, Proc. IEE, part D 127, (1980) 272.

[31] H. T. Moon, P. Huerre, L. G. Redekopp, Transitions to chaos in the Ginzburg-Landau equation, Physica D 7 (1983) 135.

[32] P. Le Gal, Complex demodulation applied to the transition to turbulence of the flow over a rotating disk, Phys. Fluids A 4 (11) (1992) 2523.

[33] R. J. Lingwood, Absolute instability of the Ekman layer and related rotating flows, J. Fluid Mech. $314(1995) 373$. 